\title{
BMJ Open Exploring linkages between climate change and sexual health: a scoping review protocol
}

\author{
Carmen H Logie (1) , ${ }^{1,2}$ Danielle Toccalino (1) , ${ }^{3}$ Anna Cooper Reed, ${ }^{2}$ \\ Kalonde Malama (D) , ${ }^{2}$ Peter A Newman (D) , ${ }^{2}$ Sheri Weiser (D) , ${ }^{4}$ Orlando Harris, ${ }^{5}$ \\ Isha Berry, ${ }^{6}$ Adebola Adedimeji ${ }^{7}$
}

To cite: Logie $\mathrm{CH}$, Toccalino D, Reed AC, et al. Exploring linkages between climate change and sexual health: a scoping review protocol. BMJ Open 2021;11:e054720. doi:10.1136/ bmjopen-2021-054720

- Prepublication history and additional supplemental material for this paper are available online. To view these files, please visit the journal online (http://dx.doi.org/10.1136/ bmjopen-2021-054720)

Received 21 June 2021 Accepted 30 September 2021

Check for updates

(C) Author(s) (or their employer(s)) 2021. Re-use permitted under CC BY-NC. No commercial re-use. See rights and permissions. Published by BMJ.

For numbered affiliations see end of article.

Correspondence to Professor Carmen H Logie; carmen.logie@utoronto.ca

\section{ABSTRACT}

Introduction The effects of climate change and associated extreme weather events (EWEs) present substantial threats to well-being. EWEs hold the potential to harm sexual health through pathways including elevated exposure to HIV and other sexually transmitted infections (STIs), disrupted healthcare access, and increased sexual and gender-based violence (SGBV). The WHO defines four components of sexual health: comprehensive sexuality education; HIV and STI prevention and care; SGBV prevention and care; and psychosexual counselling. Yet, knowledge gaps remain regarding climate change and its associations with these sexual health domains. This scoping review will therefore explore the linkages between climate change and sexual health.

Methods and analysis Five electronic databases (MEDLINE, EMBASE, PsycINF0, Web of Science, CINAHL) will be searched using text words and subject headings (eg, Medical Subject Headings (MeSH), Emtree) related to sexual health and climate change from the inception of each database to May 2021. Grey literature and unpublished reports will be searched using a comprehensive search strategy, including from the WHO, World Bank eLibrary, and the Centers for Disease Control and Prevention. The scoping review will consider studies that explore: (a) climate change and EWEs including droughts, heat waves, wildfires, dust storms, hurricanes, flooding rains, coastal flooding and storm surges; alongside (b) sexual health, including: comprehensive sexual health education, sexual health counselling, and HIV/STI acquisition, prevention and/or care, and/or SGBV, including intimate partner violence, sexual assault and rape. Searches will not be limited by language, publication year or geographical location. We will consider quantitative, qualitative, mixed-methods and review articles for inclusion. We will conduct thematic analysis of findings. Data will be presented in narrative and tabular forms.

Ethics and dissemination There are no formal ethics requirements as we are not collecting primary data. Results will be published in a peer-reviewed journal and shared at international conferences.

\section{BACKGROUND}

Climate change is one of the greatest threats to health and well-being in the 21 st century. ${ }^{12}$

\section{Strengths and limitations of this study}

- This scoping review will offer an innovative and comprehensive approach to climate change and associations with multiple sexual health domains, including sexual health education, HIV/STI prevention and treatment.

- The search strategy includes five databases and grey literature with no limitations on date, location or language to maximise the ability to find all relevant literature.

- As terminology for climate change and extreme weather events is evolving and connected to the larger planetary health field, newly emerging terms may not be included in our search.

- The five databases identified and searched for this review were chosen based on their relevance and potential coverage of the topics of interest; however, peer-reviewed literature that is not captured by these databases may be missed.

- We acknowledge that despite our best efforts to identify relevant global organisations reporting on this intersection and develop a comprehensive grey literature search, we may miss relevant documentation.

Warming temperatures, heat extremes, floods, droughts, wildfires, increased frequency of intense storms, and other extreme weather events are linked to climate change, and have impacts on human health and well-being. ${ }^{3}$ Direct and indirect health effects of extreme weather events include malnutrition, poverty, poor mental health, non-communicable diseases and infectious diseases. ${ }^{4}$ Emerging literature signals the importance of attending to the linkages between climate change and sexual health outcomes. ${ }^{56}$ HIV is an illustrative example, with prevalence highest in sub-Saharan Africa, a region that is highly impacted by climate change and associated extreme weather events such as drought, ${ }^{17-9}$ as well as Caribbean contexts such as Haiti. ${ }^{10-12}$ 
Climate change impacts broader environmental systems to increase strain on global food and water supplies. ${ }^{2}{ }^{13} 14$ Drought can increase food insecurity ${ }^{15-17}$ and migration, ${ }^{18-20}$ and disrupt both provision of-and access to-health services. ${ }^{13}$ Food insecurity is associated with HIV acquisition risks through behavioural (eg, transactional sex) and mental health (eg, alcohol use, depression) pathways. ${ }^{21} 22$ Food insecurity has also been associated with increased sexual risk practices among HIV-positive youth in sub-Saharan Africa ${ }^{23}$ and increased transactional sex and reduced safer sex efficacy among HIV-positive women. ${ }^{24}$ It has been linked with reduced condom efficacy among adolescents ${ }^{25}$ and reduced condom use with adults. ${ }^{26}$ Drought is also linked with HIV vulnerabilities, largely through reduced income. ${ }^{9}$ Water insecurity and linkages with HIV acquisition and other sexual health outcomes are understudied, ${ }^{27}$ as is the nexus of climate change, food and water insecurity, and sexual health outcomes. ${ }^{5}$

Both HIV and other sexually transmitted infections (STIs), as well as climate change and extreme weather events, disproportionately affect marginalised persons, including adolescent girls and young women, migrants, sexually and gender diverse persons, and persons living in poverty. ${ }^{68-33}$ For instance, Burke et at found that in a study of 2000 people across 19 sub-Saharan African countries, a rainfall shock (annual rain less than the 15th percentile of the local area's historical rainfall distribution) was associated with an $11 \%$ increase in HIV prevalence among rural populations who had a high HIV prevalence at baseline. In another study in Lesotho, a drought in the past 2 years was linked with higher HIV prevalence among adolescent girls in rural areas, but not among boys. ${ }^{8}$ Baker modelled the effects of climate change using datasets including 400000 persons across 25 sub-Saharan African countries, specifically examining impacts of long-term temperature increases on HIV prevalence. ${ }^{34}$ Warmer time periods were associated with increased HIV prevalence, and this increase was higher in rural regions and with younger age groups, and may be linked with men's migration and engagement in transactional sex. ${ }^{34}$ The effects of climate change are often unequal and disproportionately impact populations who have contributed the least to the crisis. ${ }^{2} 3536$ The 2020 Lancet Countdown report indicates that climate change and its contribution to extreme weather events and rising sea levels will increase human migration and displacement, both of which pose risks to human health, ${ }^{2}$ including sexual health. ${ }^{37} 38$

An ecosocial theoretical approach considers how hazardous environments, poverty and social inequities are embodied. ${ }^{39}$ The resource scarcity framework conceptualises shared structural drivers as including social (eg, gender norms), economic (eg, poverty), and ecological (eg, climate change) factors that contribute to water and food insecurity and ultimately to well-being. ${ }^{40}{ }^{41}$ Several conceptual frameworks have linked ecosocial factors to HIV and STI vulnerabilities and other sexual health concerns. Tallman et $a t^{42}$ applied the ecosyndemic approach to understand the ways through which building dams and highways in tropical forests in Brazil and Peru was linked with vector-borne infections, sex work and increased STIs. The ecosyndemics framework examines the ways in which largely human-caused environmental changes interact with economic and social inequities to harm health. ${ }^{43-45}$ Mapping ecosyndemic risk hotspots and community engagement can provide insight into how social and ecological system changes interact to shape STI and other health concerns.

Lieber et at conceptualised four paths from climate change to HIV and AIDS, including increased migration, infectious diseases, infrastructure erosion and food insecurity. For instance, extreme weather events such as drought and flooding have been linked with elevated sexual risk practices including condomless and transactional $\operatorname{sex}^{8}{ }^{86}$ and early sexual debut. ${ }^{9}$ Extreme weather events may also result in long-term and short-term migration $^{47-50}$ that is associated with increased sexual networks and sexual exploitation. ${ }^{51}{ }^{52}$ Infectious diseases, such as malaria, dengue, chikungunya and Lyme disease, may increase in prevalence and distribution due to changes in season duration, rainfall and rising temperatures. ${ }^{53-55}$ For people living with HIV, who may be immunocompromised, such infectious diseases could become more harmful and contribute to poorer HIV outcomes. ${ }^{56-58}$ Infrastructure erosion can also compromise healthcare access. Extreme weather events may disrupt HIV testing and prevention access such as in post-cyclone, ${ }^{59}$ hurricane $^{60}$ and earthquake settings. ${ }^{61}$ Food insecurity, as described earlier, has complex links with HIV acquisition risks and is exacerbated among persons living with HIV. $^{2162}$

In another recent framework, Women Deliver conceptualised paths from climate change to sexual and reproductive health outcomes, including disruptions in access to health services, as described above, as well as increased exposure to sexual and gender-based violence (SGBV) ${ }^{6}$ Extreme weather events such as drought and flooding have been linked with SGBV ${ }^{63}$ For instance, drought is associated with physical and sexual violence in 19 sub-Saharan African countries-effects are exacerbated for adolescent girls. ${ }^{63}$ Conflict arising when women have insufficient water to complete household tasks can contribute to intimate partner violence (IPV), ${ }^{4164}$ and women may also be at greater risk of non-partner violence while collecting water. ${ }^{65}$ Food insecurity is also linked with IPV $^{66}{ }^{67}$ In addition to sexual violence's direct HIV risks, ${ }^{68}$ SGBV increases HIV acquisition risks via trauma-related sequelae ${ }^{69}$ such as lower condom efficacy and coping-related substance use..$^{70} 71$

These conceptual frameworks signal the urgent need to understand and address climate change and its impacts on sexual health, which is a core component of overall health and well-being. ${ }^{72}{ }^{73}$ Sexual health is multifaceted, described by the WHO as:

A state of physical, emotional, mental and social wellbeing in relation to sexuality; it is not merely the 
absence of disease, dysfunction or infirmity. Sexual health requires a positive and respectful approach to sexuality and sexual relationships, as well as the possibility of having pleasurable and safe sexual experiences, free of coercion, discrimination and violence (p3). ${ }^{72}$

Four components of sexual health conceptualised by the WHO include: comprehensive sexuality education and information; SGBV prevention, support and care; preventing and controlling HIV infection and other STIs; and addressing sexual function and psychosexual counselling. ${ }^{72} 73$ There appears to be a dearth of scoping reviews that systematically map the evidence on climate change and related processes, and sexual health outcomes, as conceptualised by the WHO. ${ }^{72} 73$

Scoping review approaches hold particular relevance in the field of climate change and health-including providing an overview of evidence, assessing types of evidence, exploring how key concepts are defined and conceptualised in the literature, and identifying knowledge gaps. ${ }^{745}$ We identified three relevant scoping reviews on climate change and sexual health, yet none applies both a comprehensive definition of climate change and sexual health for persons of all genders. First, Thurston et al examined natural hazards, disasters, and violence against women and girls in a mixed-methods systematic review. ${ }^{76}$ This review found that natural disasters-caused by climatological, geophysical, hydrological and meteorological hazards - were associated with violence against women and girls in diverse contexts. ${ }^{76}$ While Thurston et $a l$ included climate change as a hazard, the other dimensions of sexual health beyond SGBV were not included, and their review was limited to women and girls. Second, Fatema et al published a systematic review protocol on women's health-related vulnerabilities in natural disasters, which focused on physical and psychological health and sexual harassment. ${ }^{77}$ While of importance, this review was also limited to women and girls and did not focus on the full spectrum of sexual health outcomes. Third, a recent narrative synthesis by Loewen et al examined natural disasters and disease epidemics (eg, Ebola) and related sexual and reproductive health outcomes in low/middle-income countries (LMICs). ${ }^{78}$ This review only included studies with a health outcome focus on contraception and/or abortion services ${ }^{78}$; while sexual and reproductive health are interconnected, the WHO differentiates reproductive health (abortion care, contraception), which was measured in Loewen $e t$ l s review, from sexual health (eg, HIV and STI) ${ }^{72} 73$ The review focused on acute disruptions, excluding protracted crises such as drought. Loewen et al included 13 studies in the review, and the sub-Saharan Africa studies $(n=8)$ focused on Ebola outbreaks while the other studies in Asia and the Caribbean examined natural disasters (earthquake, flood) ${ }^{78}$ Loewen et al found that structural damages to health systems resulted in access barriers; they additionally found an overall decrease in contraception (including male condom use) and family planning use in the midst of, and directly following, disruptive events. ${ }^{78}$

Our scoping review will build on these prior reviews to address knowledge gaps in how the wide-ranging effects of climate change, both acute and protracted, affect the range of sexual health outcomes among all genders and global contexts. Specifically, it will address the following research question: what is known in the literature about the associations between climate change and sexual health?

\section{METHODS AND ANALYSIS}

This scoping review explores the linkages between climate change and sexual health using the framework developed by Arksey and O'Malley ${ }^{79}$ and further developed by the Joanna Briggs Institute. ${ }^{7580}$ The reporting for this review will also follow the guidelines outlined in the Preferred Reporting Items for Systematic Review and Meta-Analysis extension for scoping reviews (PRISMA-ScR) ${ }^{81} 82$

\section{Defining sexual health}

The search strategy and eligibility criteria pertaining to sexual health were informed by the WHO's framework for operationalising sexual health. ${ }^{72} 73$ The WHO identifies sexual and reproductive health as distinct but interrelated concepts. While sexual health may encompass a person's fertility capacity and status, it also includes periods in advance of and following a person's reproductive years. ${ }^{73}$ As such, we focus this review on the four sexual health intervention areas from the WHO framework ${ }^{72}$ : (1) comprehensive sexuality education and information (including information on sexuality and reproduction, and empowerment and skills for enacting informed choices regarding sexuality and sexual health); (2) SGBV experiences and access to prevention, support and care (this includes violence targeting any gender, including sexual and gender minorities; and physical, sexual and/ or emotional violence); (3) preventing and controlling HIV infection and other STIs (this includes oral, anal and vaginal transmission; STIs including gonorrhoea, syphilis, HIV, chlamydia and herpes simplex virus; and access to testing, prevention and treatment); and (4) sexual function and psychosexual counselling (including addressing psychological, physical, physiological, and interpersonal factors associated with sexual concerns and sexual satisfaction). ${ }^{72}$ The search strategy specifically includes search terms related to HIV in addition to those related to STIs more broadly, as is outlined in the framework.

\section{Defining climate change: extreme weather events}

The search strategy and eligibility criteria pertaining to climate change were informed by the field of planetary health $^{4}$ and the work of Bell et al on extreme weather events. ${ }^{3}$ Though there is no single definition of an extreme weather event, researchers within the field of climate change have suggested that this can be broadly defined as "climate-related events that rarely occur at a given location or have significant socioeconomic 
impacts. ${ }^{3}$ We will follow examples provided by Bell et al who identify droughts, heat waves, wildfires, dust storms, hurricanes, flooding rains, coastal flooding and storm surges as comprising extreme weather events, specifically due to their identified linkages to climate change. ${ }^{3}$

\section{Search strategy}

In consultation with a University of Toronto librarian, electronic databases were assessed for their relevance and potential coverage of the topic. After careful review, five databases were selected for the search: MEDLINE, EMBASE, PsycINFO, CINAHL and Web of Science. These will be searched for relevant articles using a search strategy including text words and subject headings (eg, $\mathrm{MeSH}$, Emtree) related to sexual health and climate change, as defined above, from the inception of each database to May 2021. These terms include:

- sexual health (including sex education, sexual health counselling, sexually transmitted infections, HIV/ AIDS); OR

- sex- and gender-based violence (including sexual, physical and emotional violence, domestic and intimate partner violence, rape); AND

- climate change, extreme weather events, natural disasters (specifically, droughts, heat waves, wildfires, dust storms, hurricanes, flooding rains, coastal flooding and storm surges).

For a complete search strategy, please see online supplemental appendix I. Searches will not be limited by language, year of publication or geographical location. Records returned from this search will be managed in EndNote and Covidence. ${ }^{83} \mathrm{~A}$ manual search of the reference lists of included studies will be conducted to identify additional literature that might not have been captured in our original search. In addition, the research team will supplement the database searches with grey literature following a comprehensive search guide developed by Godin $e t a l^{84}$ and adapted by librarians at both the University of Waterloo and the University of Toronto. ${ }^{85}$ Informed by this guide, the following relevant authorities were identified and their websites will be searched for unpublished reports and grey literature: the WHO, the World Bank eLibrary, the Centers for Disease Control and Prevention, Women Deliver, the International Planned Parenthood Association, the NAP Global Network, the Asian-Pacific Resource and Research Centre for Women, and the United Nations. As with the database search, the grey literature search will not be limited by language, year of publication or geographical location. Reference lists from identified reports will also be reviewed to identify any additional reports that may not have been captured through the initial search.

\section{Study selection}

Title and abstract screen

The screening process will be compatible with the PRISMA-ScR standards recommended for peer-reviewed publications. ${ }^{81} 82$ Articles will not be assessed for quality as is practised in systematic reviews, as this is not typically done in scoping reviews ${ }^{75}$; ; and the purpose of this scoping review is to synthesise and identify themes within the available and included literature. Following the removal of duplicates, two reviewers (reviewers include ACR, DT and KM) will independently assess all identified titles and abstracts for eligibility. Quantitative, qualitative, mixed-methods and review articles will be considered for inclusion. To be included in the full-text review, articles must discuss some facet of sexual health (including sex education, sexual health counselling, STIs) and/or SGBV (including sexual, physical and emotional violence, domestic and IPV, rape) in the context of climate change and/or extreme weather events. Articles will be excluded if their primary populations of interest are infants or children (aged $<10$ years old) or if they explore sexual health or SGBV separately from climate change or extreme weather events. Additionally, conference abstracts, protocols, books or book reviews, studies conducted in animals, as well as studies for which full-text articles are not available, will be excluded. Reviewers will review a random sample of articles to confirm a common interpretation of the above criteria. Covidence software ${ }^{83}$ will be used for screening and to monitor agreement between the two reviewers' assessments. Any differences will be resolved through discussion and consensus; if consensus cannot be reached, articles will be moved to the full-text screen for further review.

\section{Full-text screen}

The full-text screen will be completed independently by two reviewers (ACR, DT and/or KM) using the same criteria outlined above. As with the title and abstract screen, Covidence software will be used to conduct the screening and monitor agreement between the reviewers' assessments. All differences in screening will be resolved through discussion and consensus of the two reviewers, or in consultation with a third reviewer if needed. The reference lists of articles meeting the criteria for full-text review will be manually searched for additional articles relevant to the review.

\section{Data charting and synthesis}

The following data will be extracted from all included studies: study details (ie, location, duration, design, population, sample size, data source, type of extreme weather event, type of sexual health outcome) and key findings (ie, impact of extreme weather event on sexual health, duration of impact, implications). Data extraction will be completed by one reviewer (ACR, DT and/or KM) and peer reviewed by a second (ACR, DT and/or KM). Data synthesis will involve analysing the findings from identified studies using thematic analysis approaches. ${ }^{86} 87$ Studies will be grouped into the four domains of sexual health described above (comprehensive sexuality education, SGBV, HIV and STI, and psychosexual counselling). We will apply sex-based and gender-based analyses ${ }^{88}$ to explore sex/gender differences in findings as well as 
integration of sex and gender factors in included studies; this will inform a discussion of gender norms and their linkage to climate change, extreme weather events and sexual health. Where relevant, we will also identify intersectional identities ${ }^{89}$ and the sociodemographic characteristics documented in studies (including but not limited to gender, age, sexual orientation and gender identity, refugee status, income, parenthood status, LMICs, sex work), and how those are included in studies and any linkages to climate change and/or sexual health impacts. We will aggregate themes to synthesise overall findings, as well as disaggregate findings by gender, type of climate change/extreme weather event (eg, drought vs flood) and by LMIC versus high-income country. We will also examine similarities and differences between LMICs, for instance, between sub-Saharan African and Caribbean contexts. Data will be presented both in narrative and tabular forms.

\section{Patient and public involvement}

There will be no patient or public involvement in this research.

\section{ETHICS AND DISSEMINATION}

As this review involves a synthesis and presentation of available resources, it does not require ethics approval. Results will be published in a peer-reviewed journal, developed into easily disseminated infographics and shared at international conferences.

\section{DISCUSSION}

This review is unique in looking at the impacts of climate change on a range of comprehensive sexual health outcomes among diverse populations. This review will provide a thematic overview of the literature on the global impacts of climate change on sexual health, offering new insight into the types of sexual health outcomes that have been assessed, sex-based and gender-based analyses, ${ }^{88}$ and contexts that have been included or overlooked in climate change and sexual health literature. Thus, by mapping the existing evidence, this scoping review will identify knowledge gaps and research and policy priorities. For instance, this review could support existing sexual health programmes and organisations to address the impacts of climate change. By doing so, programmes can be better prepared to adapt to climate change, extreme weather events and other environmental disasters. Based on available findings, we also aim to produce regional-specific findings, both between LMIC and high-income contexts and within LMICs, to inform recommendations for practice, policy and research. Moreover, the review will identify potential gaps in the existing literature to support policymakers and researchers to develop research questions and interventions to improve the delivery of sexual health services in the context of a changing climate.
There are several limitations to this review. The terminology around climate change and extreme weather events, the key concepts in this review, is continuously evolving. It is likely that newly emerging terms are not encompassed in our search strategy, which may result in relevant literature being missed. Furthermore, though we selected the five searched databases based on their relevance and coverage of the topics of interest, there may be peer-reviewed articles not indexed by these databases that are missed by our search. To combat this, we will manually search the reference lists of included articles to identify any additional literature relevant to our review. Finally, though the inclusion of a grey literature search is a strength of this review, we acknowledge that grey literature searches are imperfect and relevant documentation may be missed.

\section{Current study status}

The search strategy was developed in collaboration with a University of Toronto librarian and has been translated for each of the five databases. All five database searches have been run and title/abstract screening is underway.

\section{Author affiliations}

${ }^{1}$ Centre for Gender and Sexual Health Equity, Vancouver, British Columbia, Canada ${ }^{2}$ Factor-Inwentash Faculty of Social Work, University of Toronto, Toronto, Ontario, Canada

${ }^{3}$ Rehabilitation Science Institute, University of Toronto, Toronto, Ontario, Canada ${ }^{4}$ Department of Medicine, University of California, San Francisco, California, USA ${ }^{5}$ Department of Community Health Systems, University of California, San Francisco, California, USA

${ }^{6}$ Dalla Lana School of Public Health, University of Toronto, Toronto, Ontario, Canada ${ }^{7}$ Department of Epidemiology and Population Health, Albert Einstein College of Medicine, Bronx, New York, USA

Twitter Peter A Newman @PeterANewman and Orlando Harris @ooharrisphd Contributors $\mathrm{CHL}$ conceptualised the study and was responsible for drafting the protocol. ACR and DT substantially contributed to drafting the protocol and led writing of the methods. They developed and refined the search strategy in collaboration with the librarian, and significantly contributed to inclusion/exclusion criteria, methods and tools. ACR, DT and KM refined the inclusion/exclusion criteria. PAN was a grant co-applicant and provided manuscript feedback. SW, $\mathrm{OH}, \mathrm{IB}$ and AA provided manuscript feedback and edits. All authors read, revised and approved the final manuscript.

Funding Social Sciences and Humanities Research Council of Canada (PI: CHL, co-applicant: PAN) (\#IDG). CHL is also supported by the Canada Research Chairs Program (\#Tier2), Canada Foundation for Innovation (\#JELF), and Ontario Ministry of Research and Innovation (\#ERA).

Competing interests None declared.

Patient consent for publication Not required.

Provenance and peer review Not commissioned; externally peer reviewed.

Supplemental material This content has been supplied by the author(s). It has not been vetted by BMJ Publishing Group Limited (BMJ) and may not have been peer-reviewed. Any opinions or recommendations discussed are solely those of the author(s) and are not endorsed by BMJ. BMJ disclaims all liability and responsibility arising from any reliance placed on the content. Where the content includes any translated material, BMJ does not warrant the accuracy and reliability of the translations (including but not limited to local regulations, clinical guidelines, terminology, drug names and drug dosages), and is not responsible for any error and/or omissions arising from translation and adaptation or otherwise.

Open access This is an open access article distributed in accordance with the Creative Commons Attribution Non Commercial (CC BY-NC 4.0) license, which permits others to distribute, remix, adapt, build upon this work non-commercially, 
and license their derivative works on different terms, provided the original work is properly cited, appropriate credit is given, any changes made indicated, and the use is non-commercial. See: http://creativecommons.org/licenses/by-nc/4.0/.

\section{ORCID iDs}

Carmen H Logie http://orcid.org/0000-0002-8035-433X

Danielle Toccalino http://orcid.org/0000-0002-8738-2894

Kalonde Malama http://orcid.org/0000-0001-7655-5916

Peter A Newman http://orcid.org/0000-0003-0444-5915

Sheri Weiser http://orcid.org/0000-0002-7807-4072

\section{REFERENCES}

1 Watts N, Adger WN, Agnolucci P, et al. Health and climate change: policy responses to protect public health. Lancet 2015;386:1861-914.

2 Watts N, Amann M, Arnell N, et al. The 2019 report of the Lancet countdown on health and climate change: ensuring that the health of a child born today is not defined by a changing climate. Lancet 2019;394:1836-78.

3 Bell JE, Brown CL, Conlon K, et al. Changes in extreme events and the potential impacts on human health. J Air Waste Manag Assoc 2018;68:265-87.

4 Whitmee S, Haines A, Beyrer C, et al. Safeguarding human health in the anthropocene epoch: report of the rockefeller foundation-lancet commission on planetary health. Lancet 2015;386:1973-2028.

5 Lieber M, Chin-Hong P, Whittle HJ, et al. The synergistic relationship between climate change and the HIV/AIDS epidemic: a conceptual framework. AIDS Behav 2021;25:2266-77.

6 Women Deliver. The link between climate change and sexual and reproductive health and rights, 2021.

7 Schooley RT. Our warming planet: is the HIV-1 infected population in the crosshairs. Top Antivir Med 2018;26:67-70.

8 Low AJ, Frederix K, McCracken S, et al. Association between severe drought and HIV prevention and care behaviors in Lesotho: a population-based survey 2016-2017. PLoS Med 2019;16:e1002727.

9 Burke M, Gong E, Jones K. Income shocks and HIV in Africa. Econ 2015;125:1157-89.

10 Delcher C, Robin EG, Pierre DM. Haiti's HIV surveillance system past, present, and future. Am J Trop Med Hyg 2020;103:1372-5.

11 Bermudez LG, Stark L, Bennouna C, et al. Converging drivers of interpersonal violence: findings from a qualitative study in posthurricane Haiti. Child Abuse Negl 2019;89:178-91.

12 Spencer N, Strobl E. Hurricanes, climate change, and social welfare: evidence from the Caribbean. Clim Change 2020;163:337-57.

13 Stanke C, Kerac M, Prudhomme C, et al. Health effects of drought: a systematic review of the evidence. PLoS Curr 2013:1-44

14 Phalkey RK, Aranda-Jan C, Marx S, et al. Systematic review of current efforts to quantify the impacts of climate change on undernutrition. Proc Natl Acad Sci U S A 2015;112:E4522-9.

15 Zhao M, Running SW. Drought-induced reduction in global terrestrial net primary production from 2000 through 2009. Science 2010;329:940-3.

16 Lobell DB, Burke MB, Tebaldi C, et al. Prioritizing climate change adaptation needs for food security in 2030. Science 2008;319:80.

17 Austin KF, Noble MD, Berndt VK. Drying climates and gendered suffering: links between drought, food insecurity, and women's HIV in less-developed countries. Soc Indic Res 2020:1-22.

18 McMichael C, Barnett J, McMichael AJ. An ill wind? climate change, migration, and health. Environ Health Perspect 2012;120:646-54.

19 Weine SM, Kashuba AB. Labor migration and HIV risk: a systematic review of the literature. AIDS Behav 2012;16:1605-21.

20 Dzomba A, Govender K, Mashamba-Thompson TP, et al. Mobility and increased risk of HIV acquisition in South Africa: a mixedmethod systematic review protocol. Syst Rev 2018;7:37.

21 Weiser SD, Young SL, Cohen CR, et al. Conceptual framework for understanding the bidirectional links between food insecurity and HIV/AIDS. Am J Clin Nutr 2011;94:1729S-39

22 The Lancet HIV. The syndemic threat of food insecurity and HIV. Lancet HIV 2020;7:e75.

23 Toska E, Pantelic M, Meinck F, et al. Sex in the shadow of HIV: a systematic review of prevalence, risk factors, and interventions to reduce sexual risk-taking among HIV-positive adolescents and youth in sub-Saharan Africa. PLoS One 2017;12:e0178106.

24 Chop E, Duggaraju A, Malley A, et al. Food insecurity, sexual risk behavior, and adherence to antiretroviral therapy among women living with HIV: a systematic review. Health Care Women Int 2017;38:927-44.
25 Logie CH, Lys CL, Mackay K, et al. Syndemic factors associated with safer sex efficacy among Northern and indigenous adolescents in Arctic Canada. Int J Behav Med 2019;26:449-53.

26 Tsai AC, Hung KJ, Weiser SD. Is food insecurity associated with HIV risk? Cross-sectional evidence from sexually active women in Brazil. PLoS Med 2012;9:e1001203.

27 Maxfield A. Testing the theoretical similarities between food and water insecurity: buffering hypothesis and effects on mental wellbeing. Soc Sci Med 2020;244:112412.

28 Denton F. Climate change vulnerability, impacts, and adaptation: why does gender matter? Gend Dev 2002;10:10-20.

29 Abid M, Schilling J, Scheffran J, et al. Climate change vulnerability, adaptation and risk perceptions at farm level in Punjab, Pakistan. Sci Total Environ 2016;547:447-60

30 Alam GMM. Livelihood cycle and vulnerability of rural households to climate change and hazards in Bangladesh. Environ Manage 2017;59:777-91.

31 Ravera F, Iniesta-Arandia I, Martín-López B, et al. Gender perspectives in resilience, vulnerability and adaptation to global environmental change. Ambio 2016;45:235-47.

32 Somera N. Que [e] R [ Y ] ING the climate debates. 2, 2009.

33 Gaard G. Ecofeminism and climate change. Womens Stud Int Forum 2015;49:20-33.

34 Baker RE. Climate change drives increase in modeled HIV prevalence. Clim Change 2020;163:237-52.

35 Francis D, Cohen G, Bhatt J, et al. How healthcare can help heal communities and the planet. BMJ 2019;365:I2398

36 Mendelsohn R, Dinar A, Williams L. The distributional impact of climate change on rich and poor countries. Environ Dev Econ 2006;11:159-78.

37 Logie $\mathrm{CH}$, Okumu M, Kibuuka Musoke D, et al. Intersecting stigma and HIV testing practices among urban refugee adolescents and youth in Kampala, Uganda: qualitative findings. J Int AIDS Soc 2021;24:e25674.

38 Singh NS, Aryasinghe S, Smith J, et al. A long way to go: a systematic review to assess the utilisation of sexual and reproductive health services during humanitarian crises. BMJ Glob Health 2018;3:e000682

39 Krieger N. Methods for the scientific study of discrimination and health: an ecosocial approach. Am J Public Health 2012;102:936-44.

40 Wutich A, Brewis A. Food and scarcity: toward a broader anthropology of resource insecurity. Curr Anthropol 2014;55:444-68.

41 Choudhary N, Brewis A, Wutich A, et al. Sub-optimal household water access is associated with greater risk of intimate partner violence against women: evidence from Nepal. J Water Health 2020;18:579-94.

42 Tallman PS, Riley-Powell AR, Schwarz L, et al. Ecosyndemics: the potential synergistic health impacts of highways and dams in the Amazon. Soc Sci Med 2020:113037.

43 Ann Herring D, Swedlund AC. Ecosyndemics: global warming and the coming plagues of the twenty-first century. In: Plagues and epidemics: infected spaces past and present, 2014.

44 Singer M. Respiratory health and ecosyndemics in a time of globa warming. Health Sociology Rev 2013;22:98-111.

45 Ramírez I, Lee J, Grady S. Mapping multi-disease risk during El Niño: an ecosyndemic approach. Int J Environ Res Public Health 2018;15:2639.

46 Robinson J, Yeh E. Transactional sex as a response to risk in Western Kenya. Am Econ J Appl Econ 2011;3:35-64.

47 Myers N. Environmental refugees: a growing phenomenon of the 21st century. Philos Trans R Soc Lond B Biol Sci 2002;357:60913:609-13.

48 Marchiori L, Maystadt J-F, Schumacher I. The impact of weather anomalies on migration in sub-Saharan Africa. J Environ Econ Manage 2012;63:355-74.

49 Call MA, Gray C, Yunus M, et al. Disruption, not displacement: environmental variability and temporary migration in Bangladesh. Glob Environ Change 2017;46:157-65.

50 Mueller V, Sheriff G, Dou X, et al. Temporary migration and climate variation in eastern Africa. World Dev 2020;126. doi:10.1016/j. worlddev.2019.104704. [Epub ahead of print: 2510 2019]

51 Talman A, Bolton S, Walson JL. Interactions between HIV/AIDS and the environment: toward a syndemic framework. Am J Public Health 2013;103:253-61.

52 Voeten HACM, Vissers DCJ, Gregson S, et al. Strong association between in-migration and HIV prevalence in urban sub-Saharan Africa. Sex Transm Dis 2010;37:240-3.

53 Coates SJ, Norton SA. The effects of climate change on infectious diseases with cutaneous manifestations. Int $J$ Womens Dermatol 2021;7:8-16. 
54 Flahault A, de Castaneda RR, Bolon I. Climate change and infectious diseases. Public Health Rev 2016;37:21.

55 Meason B, Paterson R. Chikungunya, climate change, and human rights. Health Hum Rights 2014;16:105-12.

56 Mason JB, Chotard S, Bailes A. Impact of drought and HIV on child nutrition in eastern and southern Africa. Food Nutr Bull 2011;32.

57 Kwenti TE. Malaria and HIV coinfection in sub-Saharan Africa: prevalence, impact, and treatment strategies. Res Rep Trop Med 2018;9:123-36.

58 Rothan HA, Bidokhti MRM, Byrareddy SN. Current concerns and perspectives on zika virus co-infection with arboviruses and HIV. $J$ Autoimmun 2018;89:11-20.

59 Pozniak A, Atzori A, Marotta C, et al. HIV continuity of care after cyclone Idai in Mozambique. Lancet HIV 2020;7:e159-60.

60 Thomas E. Vulnerabilities associated with post-disaster declines in HIV-testing: decomposing the impact of Hurricane sandy. PLoS Curr 2018.

61 Daniel C, Logie C. Contexts of risk: a photo-voice study of Haitian youth perceptions of their HIV risk. Global Social Welfare 2016;3:255-67.

62 Logie $\mathrm{CH}$, Wang $\mathrm{Y}$, Marcus $\mathrm{N}$, et al. Factors associated with the separate and concurrent experiences of food and housing insecurity among women living with HIV in Canada. AIDS Behav 2018;22:3100-10.

63 Epstein A, Bendavid E, Nash D, et al. Drought and intimate partner violence towards women in 19 countries in sub-Saharan Africa during 2011-2018: a population-based study. PLoS Med 2020;17:e1003064.

64 Wutich A, Brewis A, Tsai A. Water and mental health. WIREs Water 2020;7:e1461

65 Bisung E, Elliott SJ. Psychosocial impacts of the lack of access to water and sanitation in low- and middle-income countries: a scoping review. J Water Health 2017;15:17-30. doi:10.2166/wh.2016.158

66 Hatcher AM, Weiser SD, Cohen CR, et al. Food insecurity and intimate partner violence among HIV-positive individuals in rural Kenya. Am J Prev Med 2021;60:563-8.

67 Hatcher AM, Stöckl H, McBride R-S, et al. Pathways from food insecurity to intimate partner violence perpetration among PeriUrban men in South Africa. Am J Prev Med 2019;56:765-72.

68 Logie $\mathrm{CH}$, Kaida A, de Pokomandy A, et al. Prevalence and correlates of forced sex as a self-reported mode of HIV acquisition among a cohort of women living with HIV in Canada. $J$ Interpers Violence 2020;35:5028-63.

69 Shamu S, Shamu P, Zarowsky C, et al. Does a history of sexual and physical childhood abuse contribute to HIV infection risk in adulthood? A study among post-natal women in Harare, Zimbabwe. PLoS One 2019;14:e0198866.

70 Jones DJ, Runyan DK, Lewis T, et al. Trajectories of childhood sexual abuse and early adolescent HIV/AIDS risk behaviors: the role of other maltreatment, witnessed violence, and child gender. J Clin Child Adolesc Psychol 2010;39:667-80.

71 Richter L, Komárek A, Desmond C. Reported physical and sexual abuse in childhood and adult HIV risk behaviour in three African countries: findings from project accept (HPTN-043). AIDS Behav 2014;18:381-9.

72 World Health Organization (WHO). Sexual health and its linkages to reproductive health: an operational approach [Internet. Geneva, Switzerland: World Health Organization, 2017. https://apps.who.int/ iris/bitstream/handle/10665/258738/9789241512886-eng.pdf

73 Stephenson R, Gonsalves L, Askew I, et al. Detangling and detailing sexual health in the SDG era. Lancet 2017;390:1014-5.

74 Munn Z, Peters MDJ, Stern C, et al. Systematic review or scoping review? Guidance for authors when choosing between a systematic or scoping review approach. BMC Med Res Methodol 2018;18:143.

75 Peters MDJ, Godfrey C, Mclnerney P. Chapter 11: scoping reviews (2020 version). In: Aromataris E, Munn Z, eds. JBI manual for evidence synthesis. Joanna Briggs Institute, 2020.

76 Thurston AM, Stöckl H, Ranganathan M. Natural hazards, disasters and violence against women and girls: a global mixed-methods systematic review. BMJ Glob Health 2021;6:e004377.

77 Fatema SR, Islam MS, East L. Women's health-related vulnerabilities in natural disasters: A systematic review protocol. BMJ Open 2019;9.

78 Loewen S, Pinchoff J, Ngo TD, et al. The impact of natural disasters and epidemics on sexual and reproductive health in low- and middle-income countries: a narrative synthesis. Int J Gynecol Obstet 2021;17.

79 Arksey H, O'Malley L. Scoping studies: towards a methodological framework. Int J Soc Res Methodol 2005;8:19-32.

80 Peters MDJ, Godfrey CM, Khalil H, et al. Guidance for conducting systematic scoping reviews. Int J Evid Based Healthc 2015;13:141-6.

81 Tricco AC, Lillie E, Zarin W, et al. PRISMA extension for scoping reviews (PRISMA-ScR): checklist and explanation. Ann Intern Med 2018;169:467-73.

82 Colquhoun H. Current best practices for the conduct of scoping reviews. EQUATOR Netw, 2016.

83 Covidence - better systematic review management [Internet], 2019. Available: https://www.covidence.org/home

84 Godin K, Stapleton J, Kirkpatrick SI, et al. Applying systematic review search methods to the grey literature: a case study examining guidelines for school-based breakfast programs in Canada. Syst Rev 2015;4:138

85 Gerstein Science Information Centre. Searching the literature: a guide to comprehensive searching in the health sciences. 2021. Toronto, ON: University of Toronto Libraries, 2021. https://guides. library.utoronto.ca/c.php?g=577919\& $\mathrm{p}=4123572$

86 Braun V, Clarke V. Using thematic analysis in psychology. Qual Res Psychol 2006;3:77-101.

87 Attride-Stirling J. Thematic networks: an analytic tool for qualitative research. Qual Res 2001;1:385-405.

88 Tannenbaum C, Greaves L, Graham ID. Why sex and gender matter in implementation research. BMC Med Res Methodol 2016;16:145,-s12874-016-0247-7.

89 Logie $\mathrm{CH}$, James L, Tharao W, et al. HIV, gender, race, sexual orientation, and sex work: a qualitative study of intersectional stigma experienced by HIV-positive women in Ontario, Canada. PLoS Med 2011;8:e1001124. 